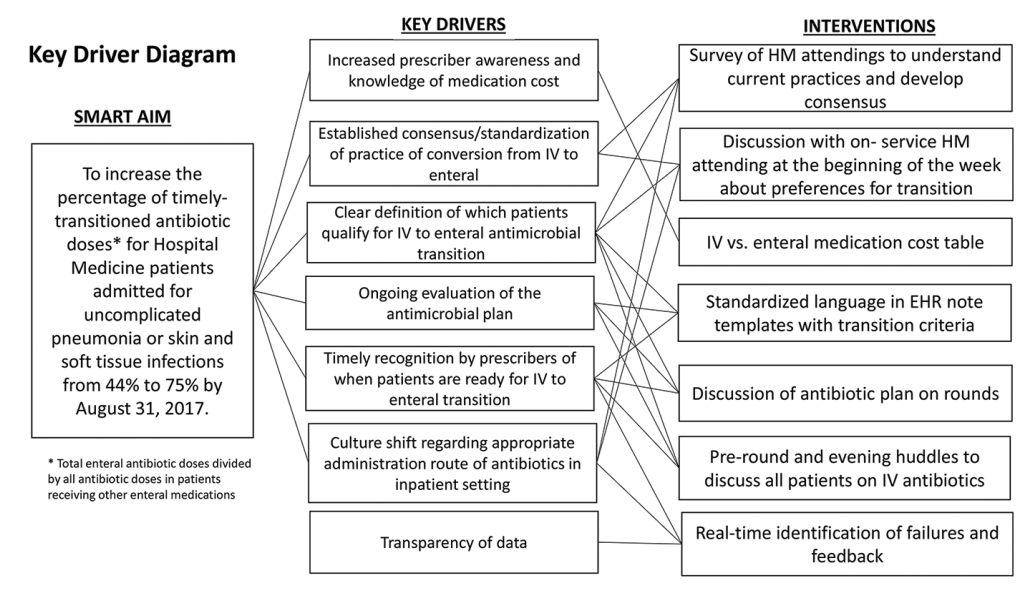

Abstract 959 Figure 1 Key driver diagram

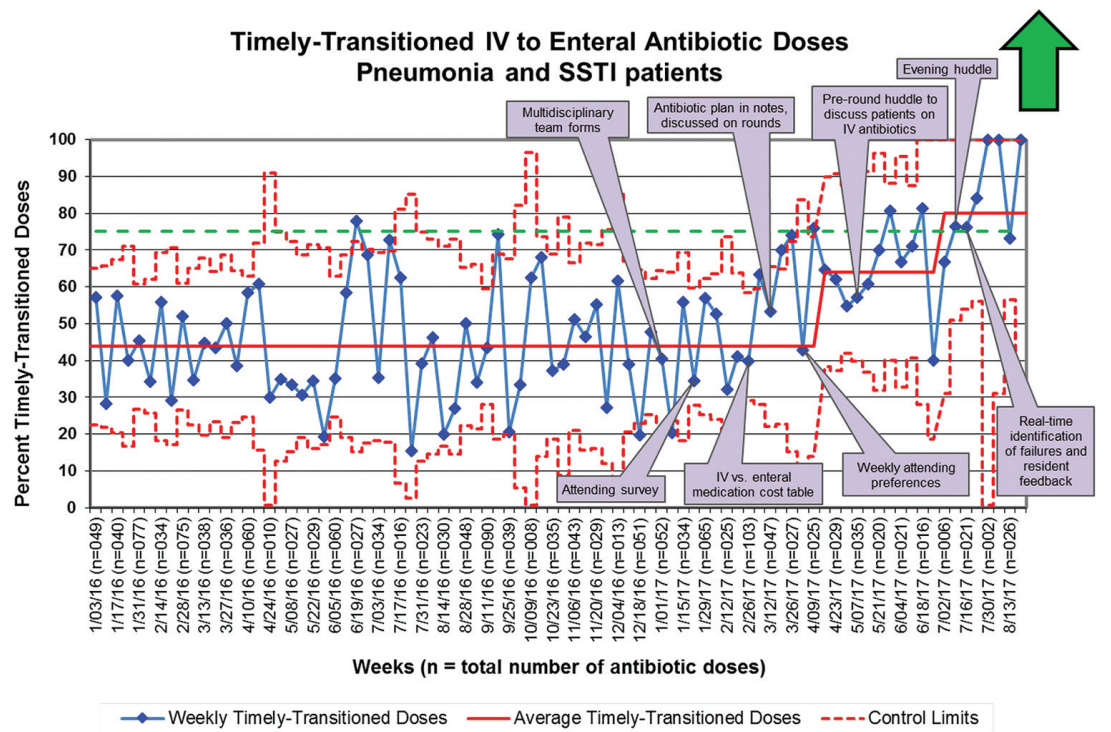

Abstract 959 Figure 2 Control chart

Conclusions Identification of clear criteria for transition from IV to enteral antibiotics can increase timely transitions for patients with uncomplicated pneumonia and SSTI.

\section{BUILDING THE BOAT WHILE SAILING: USING FORMATIVE EVALUATION TO SUPPORT PROGRAM THEORY AND IMPLEMENTATION IN THE IHI SCALE INITIATIVE}

${ }^{1}$ Abraham Wandersman, ${ }^{2}$ Soma Stout, ${ }^{3}$ Jonathan Scaccia, ${ }^{4}$ Rohit Ramaswamy, ${ }^{1}$ Kassandra Alia. ${ }^{1}$ University of South Carolina, US; ${ }^{2}$ Institute for Healthcare Improvement, US; ${ }^{3}$ HI-Faculty, US; ${ }^{4}$ University of North Carolina, US

\subsection{6/bmjoq-2017-IHI.18}

Background Spreading Community Accelerators for Learning and Evaluation (SCALE) was an 18 month initiative designed to help 24 community coalitions accelerate their health improvement work by a) applying improvement science methods and b) developing a peer-to-peer learning system to spread good ideas between communities.
Objectives We will demonstrate how formative evaluation supported and enhanced learning in SCALE. We will share how partnerships between all stakeholders and discussions on shared values facilitated co-design of methods and joint sensemaking of results.

Methods We used a multi-level, mixed method formative evaluation design grounded in the principles of empowerment evaluation and improvement science. The evaluation results were fed back to other stakeholders and processed in a timely manner to inform improvements in program design and implementation.

Results The support system (trainings and coaching) changed over time in response to evaluation data. The community coalitions reported significant progress on building improvement capability, though there were challenges in implementing and documenting outcomes from PDSA use. The majority of coalitions $(\sim 88 \%)$ made formal commitments to continue working together past the end of SCALE. As would be expected in a 24-community study, there was considerable variability in overall progress.

Conclusions Large and complex initiatives like SCALE demand much from community coalitions. Consistent with past 




Abstract 985 Figure 1 Evaluation of sexual abuse key driver diagram (KDD)

research on evaluation of health initiatives (Craig et al., 2008; Parry et al., 2013), we recommend the use of formative evaluation as means to support learning while implementation is taking place as a means to increase the likelihood of reaching outcome and making progress toward health, wellness, and equity.

\section{QUALITY IMPROVEMENT INTERVENTIONS INCREASE ADHERENCE TO GUIDELINE FOR EVALUATION AND TREATMENT OF SEXUAL ABUSE VICTIMS IN A PAEDIATRIC EMERGENCY DEPARTMENT}

Erin Hoehn, Kevin Overmann, Nafeh Fananapazir, Berkeley Bennett, Elena Duma, Eileen Murtagh Kurowski. Cincinnati Children's Hospital Medical Centre, US

10.1136/bmjoq-2017-IHI.19
Background Appropriate medical care of sexual abuse victims who present to the paediatric emergency department (PED) is vital to facilitate forensic evidence collection and prevent pregnancy and sexually transmitted infections. Despite recommendations from the American Academy of Paediatrics and Centres for Disease Control, adherence to guidelines remains low.

Objectives We aimed to increase the proportion of patient encounters at a PED for reported sexual abuse that receive guideline-adherent care from $57 \%$ to $90 \%$ within 12 months. Methods Our team of PED and child abuse paediatricians constructed a key driver diagram to outline our theory for improvement (Figure 1). Multiple plan-do-study-act cycles were conducted to test interventions aimed at key drivers, including construction of a best practice algorithm, targeted clinician education, and integration of an electronic order set. Our primary outcome was the proportion of patient



Abstract 985 Figure 2 Proportion of encounters for alleged sexual abuse with guideline-adherent care July 2015 - July 2017 